\title{
Tunable drug loading and release from polypeptide multilayer nanofilms
}

\author{
Bingbing Jiang' \\ Bingyun $\mathrm{Li}^{1,2,3}$ \\ 'Biomaterials, Bioengineering \\ and Nanotechnology Laboratory, \\ Department of Orthopaedics, School \\ of Medicine, West Virginia University, \\ Morgantown, WV, USA; ${ }^{2}$ WVNano \\ Initiative, WV, USA; ${ }^{3}$ Department \\ of Chemical Engineering, College \\ of Engineering and Mineral Resources, \\ West Virginia University, Morgantown, \\ WV, USA
}

Correspondence: Bingyun Li

Biomaterials, Bioengineering, and Nanotechnology Laboratory, Department of Orthopaedics, School of Medicine, West Virginia University, Robert C Byrd Health Sciences Center, Morgantown, WV 26506-9196, USA

Tel +I 3042931075

Fax +I 3042937070

Email bli@hsc.wvu.edu

URL http://www.hsc.wvu.edu/som/ortho/ nanomedica-group/

\begin{abstract}
Polypeptide multilayer nanofilms were prepared using electrostatic layer-by-layer self-assembly nanotechnology. Small charged drug molecules (eg, cefazolin, gentamicin, and methylene blue) were loaded in polypeptide multilayer nanofilms. Their loading and release were found to be $\mathrm{pH}$-dependent and could also be controlled by changing the number of film layers and drug incubation time, and applying heat-treatment after film formation. Antibioticloaded polypeptide multilayer nanofilms showed controllable antibacterial properties against Staphylococcus aureus. The developed biodegradable polypeptide multilayer nanofilms are capable of loading both positively- and negatively-charged drug molecules and promise to serve as drug delivery systems on biomedical devices for preventing biomedical device-associated infection, which is a significant clinical complication for both civilian and military patients. Keywords: polypeptide, self-assembly, polyelectrolyte multilayer, nanofilm, charged molecule, tunable release
\end{abstract}

\section{Introduction}

Biomedical devices are indispensable in the care of patients. However, biomedical device-associated infection is a significant clinical complication. In orthopedics, infection prevention is one of the major goals of injury management. Infection rates are $7 \%-9 \%$ for elbow replacements and $1 \%-2 \%$ for hip replacements, ${ }^{1}$ and patients with open fractures have a high risk of infection due to bacterial contamination and soft tissue damage. The incidence of Gustilo grade III open fractures may exceed $30 \%^{2,3}$ and $2 \%-15 \%$ (or higher) of combat-related extremity injuries with developed osteomyelitis. ${ }^{4}$ To reduce the risk of biomedical device-associated infection, attention has turned recently to developing drug-containing films on biomedical devices, ${ }^{5-10}$ as such films can enhance the device's specific functions including fighting infection and promoting wound-healing. ${ }^{5,7,10}$ For instance, dip coating, ${ }^{8}$ spin coating, ${ }^{9}$ spray coating, ${ }^{6}$ and covalent conjugation of antibiotics ${ }^{10}$ have been developed to prepare antimicrobial films on biomedical devices. Much effort has been devoted to controlling drug release via manipulating the dissolution or degradation of the films.

More recently, electrostatic layer-by-layer self-assembly nanotechnology has been developed ${ }^{11}$ and used to construct polyelectrolyte micro- or nanocapsules ${ }^{12,13}$ and multilayer films ${ }^{14,15}$ for drug delivery. Certain drug molecules, such as active proteins, enzymes, nucleic acids, and DNA, have been immobilized into polyelectrolyte multilayer films. The advantages of polyelectrolyte multilayer films as drug delivery systems include: (i) drug molecules can act as either functional drugs or components of the film, and they can also form a stable crosslinking structure with other film component(s) via multivalent interactions (eg, electrostatic or hydrogenbonding interactions), (ii) sustained drug release is possible through controlling the film properties, ${ }^{16}$ (iii) polyelectrolyte multilayer films have the potential to protect 
drug molecules from losing their biological functions, ${ }^{17-21}$ and (iv) the film preparation process is simple and can be automated. ${ }^{11,14-17}$,

The drug release behavior of polyelectrolyte multilayer films depends on the permeability, the disassembly or erosion of the multilayer structure, and other experimental variables. A variety of polyelectrolyte multilayer films has been studied to control drug release via ionic strength, temperature, $\mathrm{pH}$, enzyme, and hydrolytical degradation, ${ }^{12,13}$ Hayne and colleagues have bonded a thiol-bearing molecule, 5,5'-dithiobis(2-nitrobenzoic acid) (DTNB), into multilayers and 2-nitro-5-thiobenzoate dianions were released from the films by the breakdown of disulfide bonds between the DTNB and one of the film components. ${ }^{22}$ Rubner and colleagues have loaded Ketoprofen or cytochalasin D into polyelectrolyte multilayers and have shown unique zero-order drug release over a period of a few days. ${ }^{23,24}$ Caruso and Quinn have developed thermo-responsive multilayers containing poly(N-isopropylacrylamide-co-acrylic acid) and have achieved sustained drug release. ${ }^{16}$ In addition, hydrogels and micelles have been introduced into polyelectrolyte multilayers as "drug containers" to manipulate drug-loading capacity. ${ }^{25-27}$ However, only a few studies are reported on controlling the loading and release of small drug molecules. ${ }^{22,28-30}$ It is still challenging to achieve a controllable release of small charged drug molecules, probably due to the weak interactions between small drug molecules and the film components. All of these have limited the applications of polyelectrolyte multilayer films for controlled drug release, especially on biomedical devices. Introducing "binding-sites" with tunable properties within nanofilms could be very useful in achieving controllable drug loading and release in polyelectrolyte multilayer films on biomedical devices.

In this work, we prepared polypeptide multilayer nanofilms using weak polyelectrolytes of poly-L-lysine (PLL) and poly-L-glutamic acid (PLGA), and we studied the loading and release behavior of small charged drug molecules. One advantage of such biodegradable drug release systems is that drug "binding-sites" within the multilayer nanofilms can be created and tuned simply by immersing the multilayer nanofilms fabricated at one $\mathrm{pH}$ into an aqueous solution of a different $\mathrm{pH}$. For instance, PLL/PLGA multilayer nanofilms were prepared at $\mathrm{pH} 4.0$, and positivelycharged drugs including gentamicin and methylene blue (MB) were loaded into the multilayer nanofilms by immersing the nanofilms in a drug-containing solution of a higher $\mathrm{pH}$ (eg, $\mathrm{pH}$ 7.0). Negatively-charged drugs such as cefazolin were incorporated into nanofilms formed at $\mathrm{pH} 10.0$ by incubating the nanofilms in a cefazolin-containing solution of a lower $\mathrm{pH}$ (eg, $\mathrm{pH}$ 7.0). We showed that the loading and release of small charged (positively and negatively) drug molecules could be tuned by changing the number of film layers, the $\mathrm{pH}$ of the application environment or the $\mathrm{pH}$ of drug solutions, and applying post-preparation heat-treatment of the nanofilms. The driving force of drug loading and release from the multilayer nanofilms is mainly electrostatic interaction, attraction or repulsion, between the small charged drug molecules and the charged side-chains (binding-sites) of PLL or PLGA. Moreover, we found that polypeptide multilayer nanofilms loaded with antibiotics presented antibacterial properties against Staphylococcus aureus ( $S$. aureus). Therefore, the developed approach is promising for controlling the loading and release of small charged drug molecules and achieving drug release systems for preventing biomedical device associated infection.

\section{Materials and methods \\ Materials}

Poly-L-lysine $\left(\mathrm{M}_{\mathrm{n}}=150 \mathrm{kDa}\right), \operatorname{PLGA}\left(\mathrm{M}_{\mathrm{n}}=50 \mathrm{kDa}\right)$, $\mathrm{MB}$, gentamicin, and cefazolin were used (Sigma Aldrich, St. Louis, MO). The structures of these polymers and drugs are shown in Figure 1, where cefazolin, a widely used antibiotic, is a negatively-charged small drug molecule, and gentamicin, another common antibiotic, and MB, a dye indicator, are positively-charged small molecules. Quartz slides were purchased from SPI Supplies, Inc. (West Chester, PA), cut into $25 \mathrm{~mm} \times 10 \mathrm{~mm} \times 1 \mathrm{~mm}$, and cleaned by incubating in a piranha solution $\left(4: 1 \mathrm{H}_{2} \mathrm{SO}_{4} / \mathrm{H}_{2} \mathrm{O}_{2}\right)$ for $2 \mathrm{~h}$ at $80{ }^{\circ} \mathrm{C}$ followed by rinsing with deionized water. Stainless steel sheets were purchased (Small Parts, Inc., Miramar, FL) and cut into discs $(10 \mathrm{~mm} \times 0.25 \mathrm{~mm})$, which were ultrasonicated in a $2 \%$ sodium dodecyl sulphate (SDS) solution for $30 \mathrm{~min}$, washed in deionized water, and rinsed with an ethanol- $\mathrm{NaOH}$ solution and deionized water.

Buffer solutions in the $\mathrm{pH}$ range $4.0-10.0$ were used throughout this study. Buffer solutions of $\mathrm{pH} 7.0-10.0$ were prepared using $50 \mathrm{mM}$ glycine- $\mathrm{NaOH}$ and buffer solutions of $\mathrm{pH}$ 4.0-7.0 were prepared using $10 \mathrm{mM}$ Tris- $\mathrm{HCl}, 10 \mathrm{mM}$ $\mathrm{NaAc}$, and $130 \mathrm{mM} \mathrm{NaCl}$. Gentamicin $(10 \mathrm{mg} / \mathrm{mL})$ and MB (3 mg/mL) solutions were prepared in Tris-HCl buffer solutions of pHs 4.0, 5.0, and 7.0. Cefazolin was dissolved in the glycine- $\mathrm{NaOH}$ buffer solutions of $\mathrm{pHs} 7.0,8.0$, and 9.0 at a concentration of $10 \mathrm{mg} / \mathrm{mL}$. PLL $(1 \mathrm{mg} / \mathrm{mL})$ and PLGA (1 mg/mL) solutions were prepared by dissolving PLL and PLGA in the buffer solutions of pHs 4.0, 5.0, 7.0, 9.0 , and 10.0 . 
<smiles>CC(C)(C)N[C@@H](CCCCN)C(=O)C(C)(C)Cl</smiles>

Poly-L-lysine<smiles>CNC(CCC(=O)[O-])C(=O)C(C)(C)C</smiles>

Poly-L-(glutamic acid)<smiles>CN(C)c1ccc2nc3ccc(=[N+](C)C)cc-3sc2c1</smiles>

Methylene blue

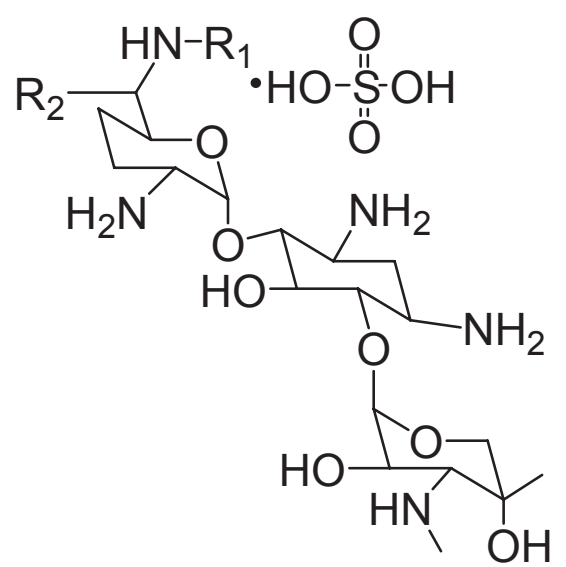

Gentamicin sulfate salt<smiles>Cc1nnc(SCC2=C(C(=O)O)N3C(=O)[C@H](NC(=O)Cn4cncn4)[C@H]3SC2)s1</smiles>

Cefazolin sodium salt

Figure I Structures of studied polypeptides and drugs.

\section{Assembly of polypeptide multilayer nanofilms}

Polypeptide multilayer nanofilms were prepared at $\mathrm{pHs} 4.0$, 5.0, 7.0, 9.0, and 10.0 using a dipping-machine (Riegler and Kirstein $\mathrm{GmbH}$, Berlin, Germany); all the aqueous media used were of the same $\mathrm{pH}$ for the same sample. In brief, pre-cleaned quartz slides or stainless steel discs were dipped in a PLL solution for $20 \mathrm{~min}$ followed by rinsing with corresponding buffer solution for $3 \mathrm{~min}$ and drying with air. The samples were then dipped in a PLGA solution for $20 \mathrm{~min}$, rinsed with buffer solution for $3 \mathrm{~min}$, and dried with air. These two dipping processes, ie, dipping in PLL and PLGA solutions, were referred to as one deposition cycle. By repeating the deposition cycle, polypeptide multilayer nanofilms, (PLL/ PLGA), were prepared where $\mathrm{n}$ is the number of deposition cycles or bilayers.

The formation of polypeptide multilayer nanofilms on quartz slides was examined using UV-vis spectrometry. Images of PLL/PLGA multilayer nanofilms were obtained using atomic force microscopy (AFM, PicoSPM ${ }^{\circledR}$ II, Tempe, AZ) operating in a tapping mode with a silicon nitride cantilever tip. The growth of multilayer nanofilms on stainless steel discs with bilayers was measured using ellipsometry (M-2000, JA Woollam Co., Lincoln, NE).

\section{Post-preparation heat-treatment of polypeptide multilayer nanofilms}

Polypeptide multilayer nanofilms, (PLL/PLGA) ${ }_{10}$ and (PLL/ PLGA $)_{20}$, on quartz slides were treated at $120^{\circ} \mathrm{C}$ in a vacuum oven (Isotemp Model 281, Fisher Scientific, Pittsburgh, PA) for $4 \mathrm{~h}$. The vacuum applied was 380 Torr.

\section{Stability of polypeptide multilayer nanofilms}

The stability of polypeptide multilayer nanofilms in aqueous media was tested. In one set of studies, (PLL/PLGA) ${ }_{20}$ films on quartz slides were assembled at pHs 4.0, 7.0, and 10.0, and then incubated in a phosphate-buffered saline (PBS) of $\mathrm{pH}$ 7.0. In another set of experiments, (PLL/PLGA) $)_{20}$ films prepared at $\mathrm{pH} 10.0$ on quartz slides were incubated in PBS solutions of pHs 4.0, 7.0, and 9.0. After incubating for $0,0.5$, $2.5,8,24,48,96$, and $192 \mathrm{~h}$, the samples were dried and their 
absorbances in the range of 190-290 nm were recorded using UV-vis spectrometry. All the data were averaged from three measurements. The absorbances of the same polypeptide multilayer nanofilm were compared between the time points studied.

\section{Antibiotic- and MB-loading in polypeptide multilayer nanofilms}

The loading of positively- and negatively-charged small drug molecules and drug models in polypeptide multilayer nanofilms was studied. The influences of number of film layers as well as the drug-loading time and drug solution $\mathrm{pH}$ were investigated. Polypeptide multilayer nanofilms prepared at pHs 4.0, 5.0, and 7.0 were used to load positively-charged drug molecules (ie, gentamicin and $\mathrm{MB}$ ), and those at $\mathrm{pHs}$ 7.0, 9.0, and 10.0 were used to load negatively-charged drug molecules (ie, cefazolin). To determine the effect of drug solution $\mathrm{pH}$ on drug loading, (PLL/PLGA) ${ }_{20}$ films prepared at $\mathrm{pH} 4.0$ were incubated in $\mathrm{MB}$ solutions of $\mathrm{pHs}$ 4.0, 5.0, and 7.0, and (PLL/PLGA) $)_{20}$ films prepared at $\mathrm{pH}$ 10.0 were immersed in cefazolin solutions of $\mathrm{pHs} 7.0,8.0$, 9.0, and 10.0 .

In general, polypeptide multilayer nanofilms on quartz slides were incubated in the corresponding drug solutions at ambient temperature. At time periods of 2, 5, 10, 20, 40, and $60 \mathrm{~min}$, the samples were rinsed with deionized water and dried with $\mathrm{N}_{2}$ gas followed by UV-vis absorbance measurements. The loading of cefazolin, gentamicin, and MB was determined by measuring the absorbance at $270 \mathrm{~nm}, 270 \mathrm{~nm}$, and $665 \mathrm{~nm}$, respectively, using UV-vis spectrometry. ${ }^{28,29}$

In order to obtain the total loading amounts of cefazolin, gentamicin, and $\mathrm{MB}$ in polypeptide multilayer nanofilms, the drug-loaded samples were ultrasonicated in $1 \mathrm{~mL}$ PBS for $30 \mathrm{~min}$, and the ultrasonication process was repeated three or more times until no peak absorbance referring to the corresponding drugs on quartz slides could be observed using UV-vis spectrometry. The drug concentration in the PBS was analyzed by recording the peak absorbance of the drugs. Raw data were converted to concentration of drug $\left(C_{n}, \mu g / m L\right)$ referring to the standard curves we obtained (data not shown). The drug released into the PBS solutions $\left(M_{n}, \mu \mathrm{g} / \mathrm{cm}^{2}\right)$ was calculated from an equation: $M_{n}=C_{n} \times V / A_{n}$, where $V$ is the total volume of the PBS and $A_{n}$ is the surface area of the nanofilms on substrates. The total drug loaded in polypeptide multilayer nanofilms was determined as the cumulative amount of drugs released during the ultrasonication processes. It is worth noting that the peak absorbance of gentamicin at $270 \mathrm{~nm}$ was observed in gentamicin-loaded polypeptide multilayer nanofilms but the absorbance of the released gentamicin in the PBS solutions was hard to detect. As a result, the actual amount of gentamicin loaded was not reported in this study.

\section{In vitro drug release from polypeptide multilayer nanofilms}

Drug-loaded polypeptide multilayer nanofilms on quartz slides were incubated in $10 \mathrm{~mL}$ PBS of a certain $\mathrm{pH}$ (eg, $\mathrm{pHs}$ 4.0, 5.0, 7.0, 9.0, or 10.0). 0.6 mL of PBS solution was taken at a certain time period and $0.6 \mathrm{~mL}$ of fresh PBS was added to keep the volume of the release medium constant. The sample solutions of cefazolin and MB were analyzed using UV-vis spectrometry by measuring their absorbance at $270 \mathrm{~nm}$ and $665 \mathrm{~nm}$, respectively. The total drug release $\left(\mu \mathrm{g} / \mathrm{cm}^{2}\right)$ was calculated as detailed before.

\section{S. aureus Kirby-Bauer disk diffusion assay}

A modified Kirby-Bauer technique was used to assess the antibacterial activity of polypeptide multilayer nanofilms loaded with antibiotics. ${ }^{31,32}$ A clinical isolate $S$. aureus was grown overnight in Mueller-Hinton broth, and the turbidity was adjusted to $0.5 \mathrm{McF}$ arland. A cotton swab was dipped in the $S$. aureus suspension and rubbed across the surface of a Mueller-Hinton blood agar plate. Cefazolin- and gentamicinloaded polypeptide multilayer nanofilms on stainless steel discs were inserted parallel to the agar plate surface. The plates were inverted and incubated at $37^{\circ} \mathrm{C}$ without shaking for $24 \mathrm{~h}$ before observation. The diameters of the zones of inhibition were measured six times from different directions, and the experiments were repeated at least three times. The average diameters of the zones were calculated.

\section{Results \\ Growth curve and stability of PLL/PLGA multilayer nanofilms}

The formation of PLL/PLGA multilayer films was examined using UV-vis spectrometry, ellipsometry, and AFM. Figure 2a shows that the absorbance of PLL/PLGA multilayer films on quartz slides increased with increasing number of bilayers. In the $\mathrm{pH}$ values studied (ie, $\mathrm{pH} 4.0,7.0$, and 10.0), the smallest increase in absorbance was observed in the multilayer films constructed at $\mathrm{pH} 7.0$. Figure $2 \mathrm{~b}$ presents the thickness of polypeptide multilayer films. One can see that the thickness growth of PLL/PLGA multilayer films increased linearly with an increasing number of deposition bilayers. Similar to the UV-vis absorbance data, the least thickness was observed in 
(a)
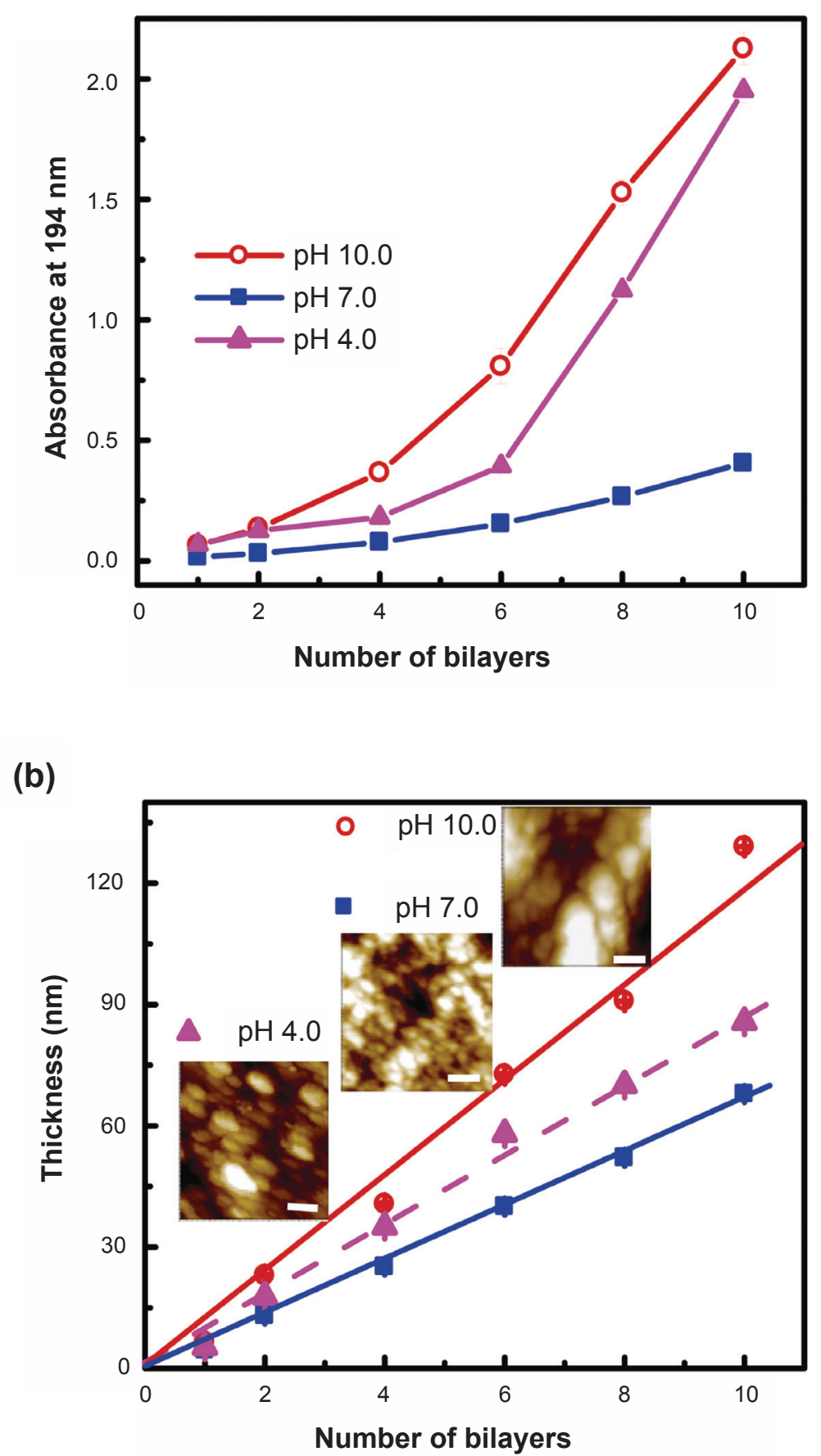

Figure 2 Growth profiles of $\mathrm{pH}$-controllable polypeptide multilayer nanofilms a) on quartz slides and examined using UV-vis spectrometry, and b) on stainless steel discs and examined using ellipsometry. The inset in b) presents the atomic force microscope images of multilayer films formed at pHs 4.0, 7.0, and I0.0. The scale bars are $200 \mathrm{~nm}$.

the multilayer films constructed at $\mathrm{pH} 7.0$ and the greatest thickness in the films prepared at $\mathrm{pH}$ 10.0. The thickness per layer of the PLL/PLGA multilayer films prepared at pHs 4.0, 7.0 and 10.0 were $4.3 \pm 0.3 \mathrm{~nm}, 3.4 \pm 0.1 \mathrm{~nm}$, and $5.9 \pm 0.2 \mathrm{~nm}$, respectively.
The surface morphology of polypeptide multilayer nanofilms formed at $\mathrm{pHs} 4.0,7.0$, and 10.0 was examined using AFM (Figure $2 b$ insets). Particulate domains were observed. The size of the particulate domains of the nanofilms prepared at $\mathrm{pH} 7.0$ was around tens of nanometers and was 
much smaller than those of the nanofilms assembled at $\mathrm{pHs}$ 4.0 and 10.0 .

One concern in developing polyelectrolyte multilayer films is their stability. Our stability studies of the PLL/PLGA nanofilms in aqueous solutions showed no obvious changes in absorbance (data not shown) in the wavelength range of 190-290 nm after more than one week at all the $\mathrm{pH}$ values studied (ie, $\mathrm{pH} 4.0,7.0$, and 9.0). This means that PLL/PLGA multilayer nanofilms are stable and can tolerate $\mathrm{pH}$ shifts in our drug loading and release processes.

\section{Tunability of drug loading in PLL/PLGA multilayer nanofilms}

The influence of $\mathrm{pH}$ at which the multilayer nanofilms were prepared and time of incubation on drug loading was studied in (PLL/PLGA) ${ }_{20}$ nanofilms (Figure 3). It was found that the

(a)

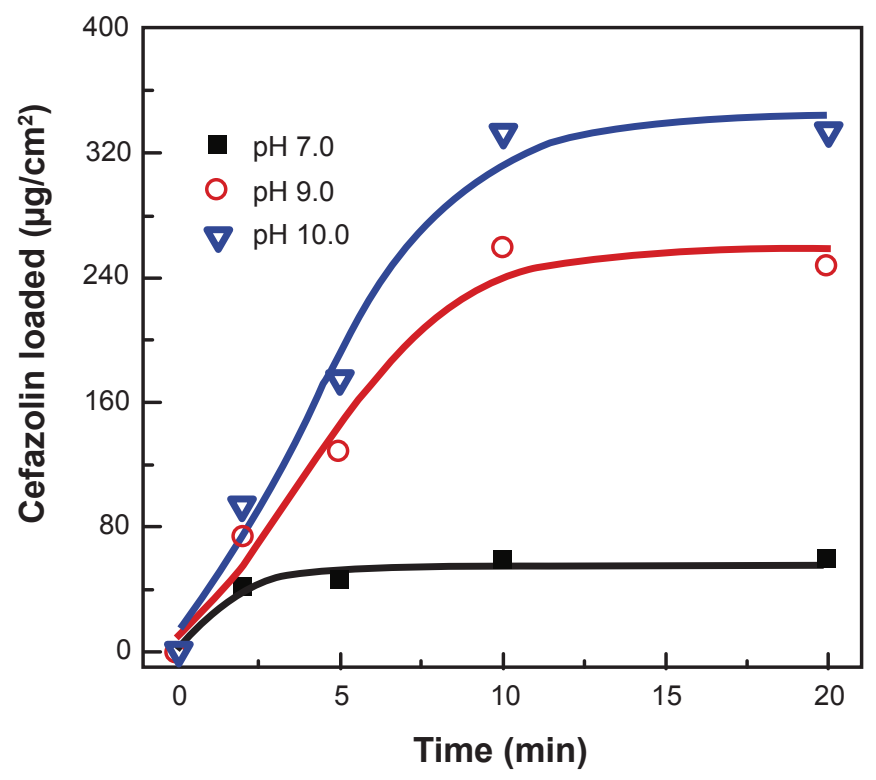

(b)

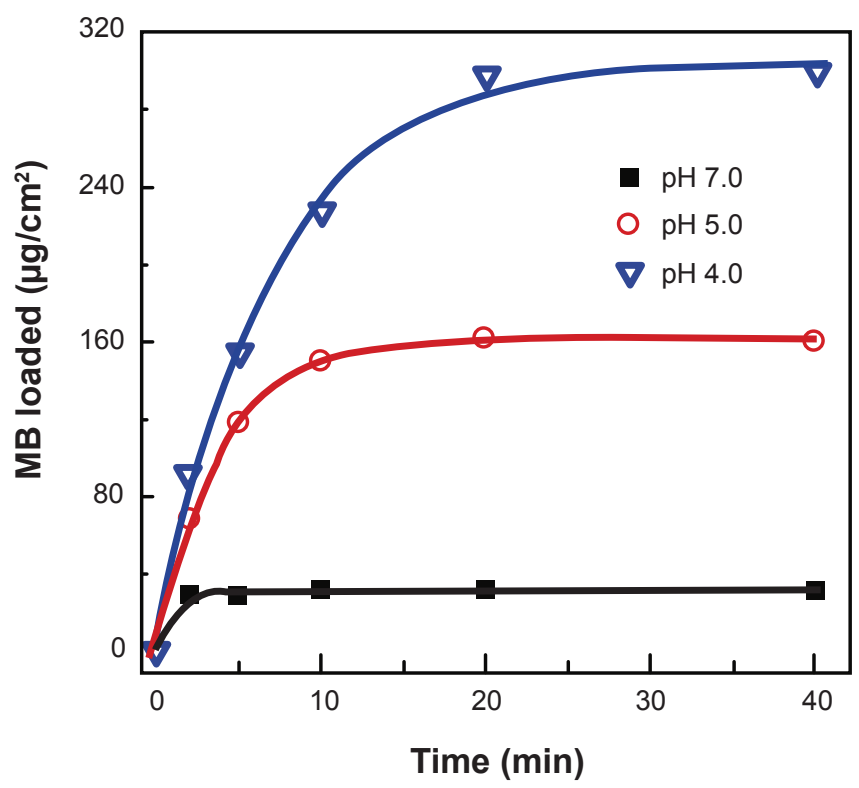

Figure 3 Effects of $\mathrm{pH}$ at which nanofilms were prepared on drug-loading profiles of a) cefazolin and $\mathbf{b})$ MB in (PLL/PLGA) ${ }_{20}$ nanofilms. The $\mathrm{pH}$ of both cefazolin and MB solutions was 7.0 .

Abbreviations: MB, methylene blue; PLGA, poly-L-glutamic acid; PLL, poly-L-lysine. 
loading of drugs increased with the incubation time and the loading of cefazolin and MB could reach their maximum loading (ie, capacity) within 10 and $20 \mathrm{~min}$, respectively. (PLL/PLGA) $)_{20}$ nanofilms formed at different $\mathrm{pHs}$ showed different drug-loading capacities. More drugs were captured in the nanofilms prepared at a $\mathrm{pH}$ away from $\mathrm{pH} 7.0$ than at $\mathrm{pH} 7.0$, and loading was faster in the nanofilms assembled at pHs 10.0 and 4.0 than at $\mathrm{pH}$ 7.0. Cefazolin-loading capacity in the nanofilms formed at $\mathrm{pH} 10.0 \mathrm{was} \sim 330 \mu \mathrm{g} / \mathrm{cm}^{2}$; it was the highest and it was almost six times that of nanofilms prepared at $\mathrm{pH} 7.0\left(\sim 60 \mu \mathrm{g} / \mathrm{cm}^{2}\right)$. Similarly, the MB-loading capacity in (PLL/PLGA) ${ }_{20}$ nanofilms assembled at $\mathrm{pH} 4.0$ was about ten times that in the nanofilms prepared at $\mathrm{pH}$ 7.0.

The influence of drug solution $\mathrm{pH}$ on drug loading was also investigated. Figure 4 shows that more cefazolin was loaded in (PLL/PLGA $)_{20}$ nanofilms at a lower $\mathrm{pH}$ in the range of $\mathrm{pH} 7.0-10.0$, and more $\mathrm{MB}$ was loaded at a higher $\mathrm{pH}$ in the range of $\mathrm{pH} 4.0-7.0$. Cefazolin-loading capacity at $\mathrm{pH} 7.0$ was about four times that at $\mathrm{pH} 9.0$. Loading of cefazolin at $\mathrm{pH} 10.0$ was not detected and the loading capacity of MB at $\mathrm{pH} 7.0$ was about sixteen times that at $\mathrm{pH}$ 4.0.

Figure 5a presents the drug-loading capacity versus bilayers of (PLL/PLGA) nanofilms. The amounts of drugs, either cefazolin or MB, loaded increased approximately linearly with an increasing number of bilayers.
The influence of post-preparation heat-treatment on drug loading is shown in Figure $5 \mathrm{~b}$. After heat-treatment at $120^{\circ} \mathrm{C}$ for $4 \mathrm{~h}$, both (PLL/PLGA) ${ }_{10}$ and (PLL/PLGA) ${ }_{20}$ nanofilms had $\sim 30 \%$ increase in drug loading.

In addition, Figure 6 shows that gentamicin was loaded into PLL/PLGA multilayer nanofilms within 20 to $40 \mathrm{~min}$, and gentamicin and MB, both positively-charged, had similar drug-loading kinetics in (PLL/PLGA) ${ }_{20}$ nanofilms.

\section{Tunability and kinetics of drug release from PLL/PLGA multilayer nanofilms}

Environmental conditions (eg, $\mathrm{pH}$ ) influenced the drug release from polypeptide multilayer nanofilms. We studied the release of cefazolin and MB from (PLL/PLGA) ${ }_{20}$ nanofilms in a wide range of $\mathrm{pH}$ values. Figure 7 shows that more cefazolin was released at a higher $\mathrm{pH}$ than at a lower $\mathrm{pH}$ in the $\mathrm{pH}$ range 7.0-10.0; the amount of cefazolin released at $\mathrm{pH}$ $10.0, \sim 340 \mu \mathrm{g} / \mathrm{cm}^{2}$, was about twice that released at $\mathrm{pH} 7.0$, $\sim 190 \mu \mathrm{g} / \mathrm{cm}^{2}$. Also, more MB was released at a lower $\mathrm{pH}$ than at a higher $\mathrm{pH}$ in the $\mathrm{pH}$ range 4.0-7.0.

Moreover, the number of film bilayers, the $\mathrm{pH}$ at which the nanofilms were prepared, and post-preparation heat-treatment influenced drug release from polypeptide multilayer nanofilms. The number of film bilayers influenced the drug release behavior. Figure 8a shows that there was a burst release in the first few

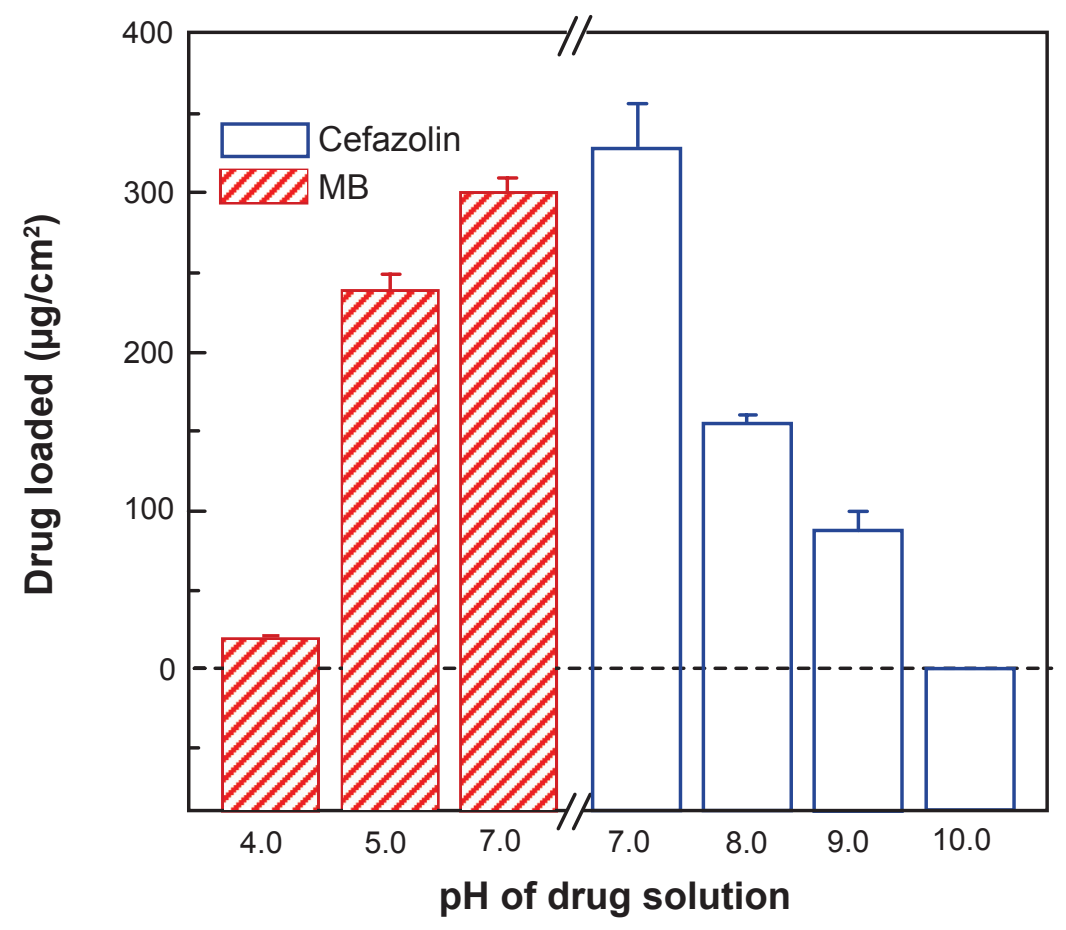

Figure 4 Effects of drug solution $\mathrm{pH}$ on drug-loading capacity of polypeptide multilayer nanofilms. Cefazolin was loaded at pHs 7.0,8.0,9.0, and I0.0 in (PLL/PLGA) ${ }_{20}$ nanofilms assembled at 10.0. MB was loaded at $\mathrm{pHs} 4.0,5.0$, and 7.0 in (PLL/PLGA) ${ }_{20}$ nanofilms prepared at $\mathrm{pH} 4.0$.

Abbreviations: MB, methylene blue; PLGA, poly-L-glutamic acid; PLL, poly-L-lysine. 

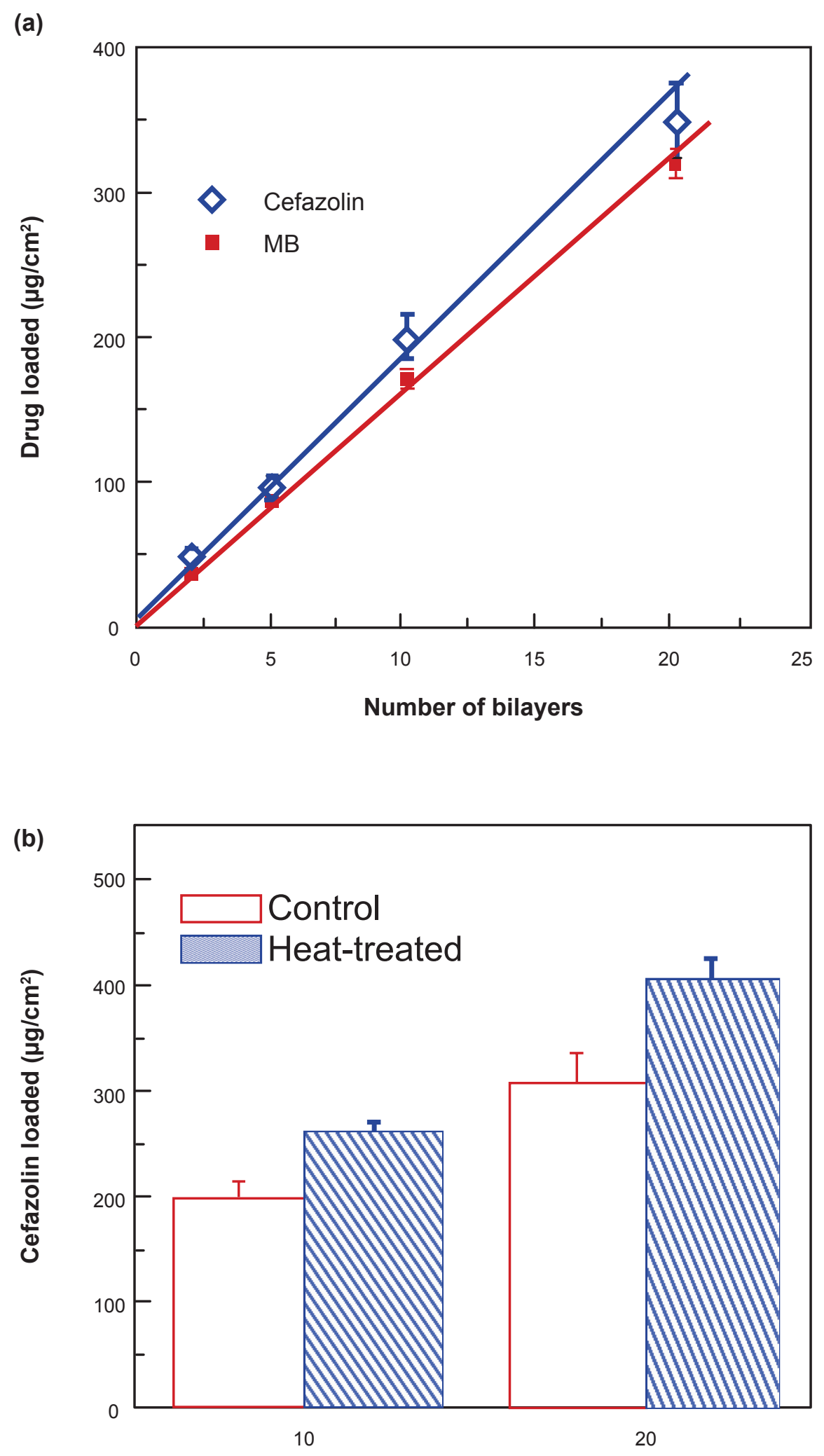

Number of bilayers

Figure 5 Effects of a) film bilayers and b) heat-treatment on drug-loading capacity. The $\mathrm{pH}$ of both cefazolin-and MB-loading solutions was 7.0. Cefazolin was loaded in (PLL/PLGA) ${ }_{20}$ nanofilms assembled at pH 10.0. MB was loaded in (PLL/PLGA) ${ }_{20}$ nanofilms prepared at pH 4.0.

Abbreviations: MB, methylene blue; PLGA, poly-L-glutamic acid; PLL, poly-L-lysine. 


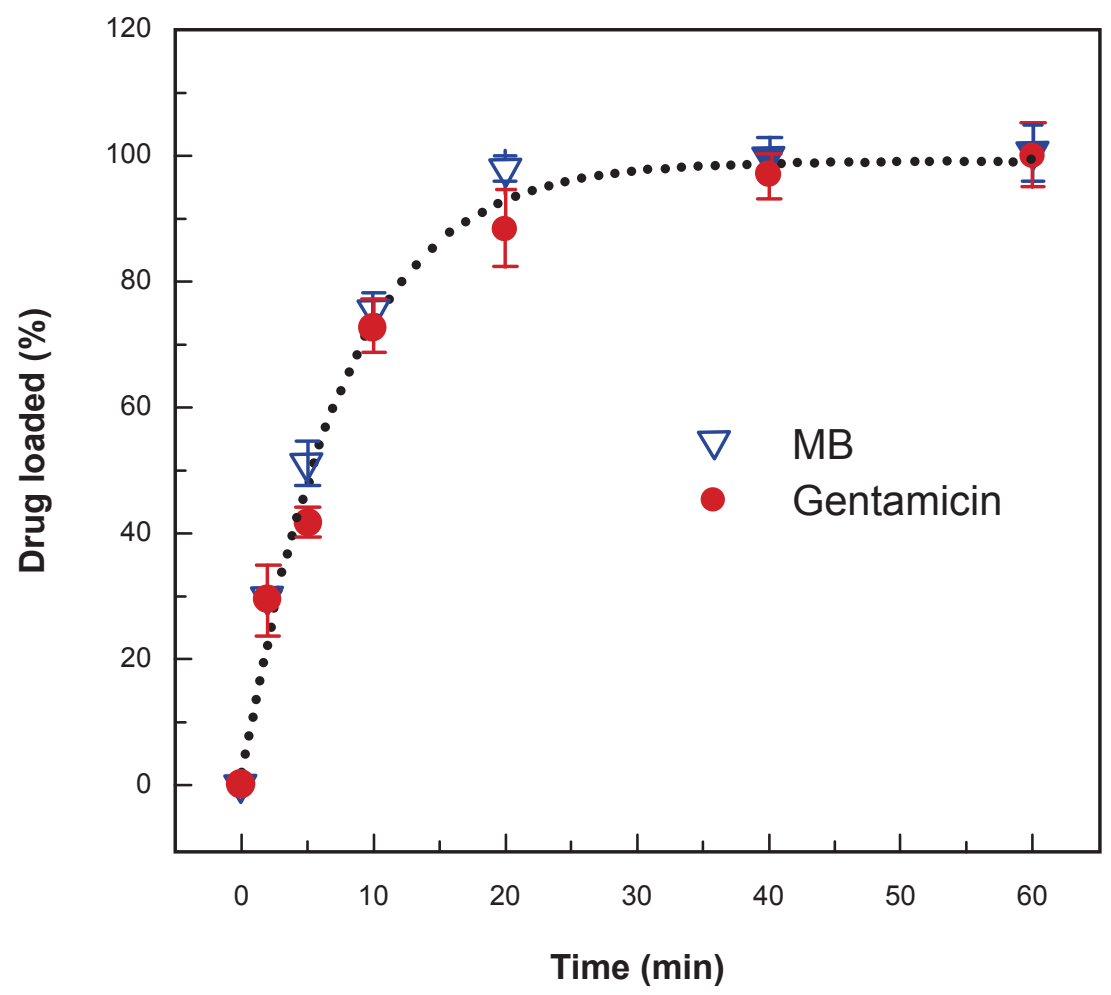

Figure 6 Loading profiles of gentamicin and MB in (PLL/PLGA) ${ }_{20}$ nanofilms assembled at $\mathrm{pH}$ 4.0. Both gentamicin and MB were loaded at $\mathrm{pH}$ 7.0. UV-vis absorbances of gentamicin at $270 \mathrm{~nm}$ and $\mathrm{MB}$ at $665 \mathrm{~nm}$ were recorded, and the highest absorbance values (ie, at $60 \mathrm{~min}$ ) were set as $100 \%$ for gentamicin and $\mathrm{MB}$.

Abbreviations: MB, methylene blue; PLGA, poly-L-glutamic acid; PLL, poly-L-lysine.

hours (mainly the first hour) in all the cefazolin-loaded samples. Up to $95 \%$ cefazolin was released from 2- and 5-bilayer PLL/ PLGA nanofilms within $48 \mathrm{~h}$, and $85 \%$ and $75 \%$ were released from the 10- and 20-bilyer PLL/PLGA nanofilms, respectively. More cefazolin was released from a higher number of bilayers of nanofilms. For instance, 50, 90, 180, and $250 \mu \mathrm{g} / \mathrm{cm}^{2}$ cefazolin were released at $48 \mathrm{~h}$ from 2, 5, 10, and 20-bilayer PLL/PLGA nanofilms, respectively. Similarly, there was a burst release in the first few hours in MB-loaded PLL/PLGA multilayer nanofilms, and more $\mathrm{MB}$ was released with an increasing number of bilayers (Figure $8 \mathrm{~b}$ ).

Figure 9 shows that the $\mathrm{pH}$ at which PLL/PLGA nanofilms were prepared also affected drug release. More cefazolin was released from (PLL/PLGA) ${ }_{20}$ nanofilms assembled at a higher $\mathrm{pH}$ in the $\mathrm{pH}$ range $7.0-10.0$, and more $\mathrm{MB}$ was released from (PLL/PLGA $)_{20}$ nanofilms deposited at a lower $\mathrm{pH}$ in the $\mathrm{pH}$ range $4.0-7.0$.

In addition, post-preparation heat-treatment influenced drug release (Figure 10). A greater amount of cefazolin was released from heat-treated samples than from untreated ones. Meanwhile, the heat-treatment slowed drug release from the PLL/PLGA nanofilms. Up to $80 \%$ of loaded cefazolin was released in the first $24 \mathrm{~h}$ from the 10- and 20-bilayer PLL/PLGA nanofilms without heat-treatment, while approximately $67 \%$ and 60\% was released from the heat-treated 10- and 20-bilayer PLL/PLGA nanofilms, respectively (Figure 10 inset).

\section{Kirby-Bauer assays}

Studies were conducted to evaluate the antibacterial effects of cefazolin- and gentamicin-loaded polypeptide multilayer nanofilms. Figure 11a shows that the average diameter of zone of inhibition increased with increasing film bilayers. The discs with cefazolin had a zone of inhibition of $18.8 \pm 0.8 \mathrm{~mm}$, $20.7 \pm 0.9 \mathrm{~mm}$, and $23.9 \pm 0.8 \mathrm{~mm}$, respectively, for 5, 10, and 20-bilayer PLL/PLGA nanofilms. The average zone diameters, for 5, 10, and 20-bilayers, of gentamicin-loaded PLL/PLGA nanofilms were $20.2 \pm 1.0 \mathrm{~mm}, 23.6 \pm 0.7 \mathrm{~mm}$, and $27.0 \pm$ $0.9 \mathrm{~mm}$, respectively. The cefazolin- and gentamicin-loaded 20-bilayer PLL/PLGA nanofilms assembled at different $\mathrm{pHs}$ also showed different antibacterial activity (Figure 11b). The average zone diameters for cefazolin-containing nanofilms assembled at $\mathrm{pH} 8.0,9.0$, and 10.0 were $18.3 \pm 0.8 \mathrm{~mm}, 20.5 \pm$ $1.0 \mathrm{~mm}$, and $23.9 \pm 0.8 \mathrm{~mm}$, respectively, and the average zone diameters were $17.5 \pm 1.1 \mathrm{~mm}, 22.5 \pm 1.0 \mathrm{~mm}$, and $27.0 \pm$ $0.9 \mathrm{~mm}$ for gentamicin-containing (PLL/PLGA) ${ }_{20}$ nanofilms deposited at $\mathrm{pH} 7.0,5.0$, and 4.0, respectively. 


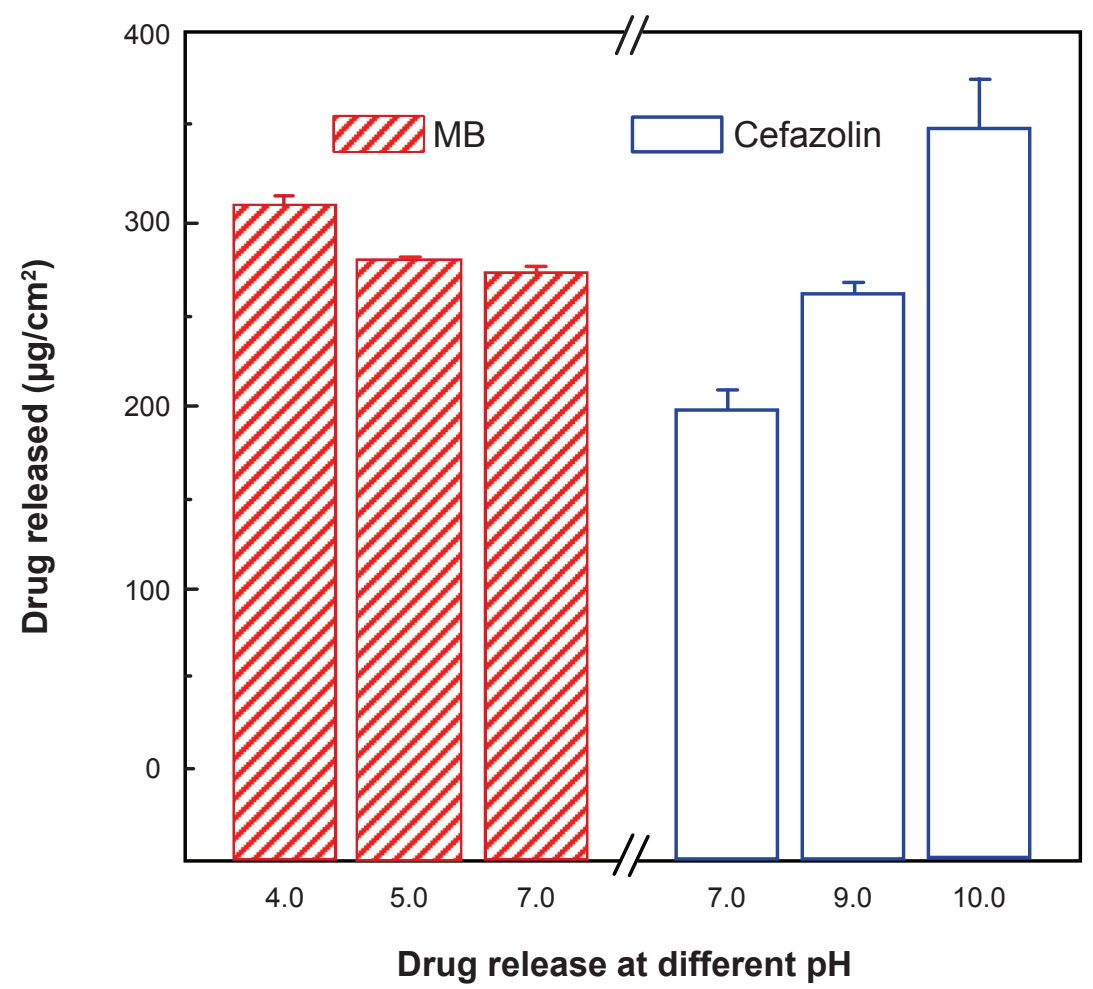

Figure 7 Effects of environmental pH on release of cefazolin and MB from polypeptide multilayer nanofilms. Cefazolin was released in PBS solutions of pHs 7.0,9.0, and I0.0 for $48 \mathrm{~h}$, and MB was released in PBS solutions of $\mathrm{pHs} 4.0,5.0$, and 7.0 for $48 \mathrm{~h}$. Cefazolin was released from (PLL/PLGA) 20 nanofilms that were assembled at pH 10.0 and loaded with cefazolin at pH 7.0 for 20 min. MB was released from (PLL/PLGA) nanofilms that were prepared at pH 4.0 and loaded with MB at pH 7.0 for 20 min.

Abbreviations: MB, methylene blue; PBS, phosphate-buffered saline; PLGA, poly-L-glutamic acid; PLL, poly-L-lysine.

\section{Discussion}

In this study, we developed biodegradable polypeptide multilayer nanofilms made of two weak polyelectrolytes as potential drug release systems on biomedical devices. Our developed polypeptide multilayer nanofilms on stainless steel discs and quartz slides possessed the capability to load both negatively- and positively-charged small drug molecules, and the drug loading and release were tunable and $\mathrm{pH}$-dependent.

\section{Mechanism of $\mathrm{pH}$-dependent drug loading and release}

Compared to strong polyelectrolytes, such as poly(dially ldimethylammonium chloride) (PDDA) and poly(styrene sulfonate) (PSS), weak polyelectrolytes such as PLL, PLGA, poly(ethyleneimine) (PEI), poly(acrylic acid) (PAA), and poly(allylamine hydrochloride) (PAH), may present various ionization statuses and surface charges as their environmental $\mathrm{pH}$ changes. At a $\mathrm{pH}$ below or above the isoelectric point (pI), a weak polyelectrolyte is partially charged and may adopt a coiled structure in polyelectrolyte multilayer nanofilms due to the decrease in charge repulsion among themselves. ${ }^{13,20}$ The coil-structured polymer contains uncharged segments that may be converted to be charged ("binding sites") if the environmental $\mathrm{pH}$ is switched to a value at which the weak polyelectrolyte becomes more ionized. As a result, binding sites within multilayers made of weak polyelectrolytes can be created for capturing oppositely-charged drug molecules.

In our study, we prepared PLL/PLGA multilayer nanofilms using electrostatic layer-by-layer self-assembly based on alternative deposition of PLL and PLGA on a substrate. Mechanisms of drug loading and release from the developed PLL/PLGA multilayer nanofilms are proposed in Figure 12. PLL and PLGA are weak polyelectrolytes; PLGA possesses a net negative charge at a $\mathrm{pH}$ higher than $\mathrm{pH}$ 2.6, the $\mathrm{pI}$ of PLGA, ${ }^{33,34}$ and PLL presents a net positive charge at a $\mathrm{pH}$ lower than its $\mathrm{pI}, \mathrm{pH} 12.5 .^{32}$ Therefore, in the multilayer films formed in an acid solution, eg, $\mathrm{pH}$ 4.0, PLGA is partially charged and adopts a coiled structure (Figure 12 top part), and its net charge decreases as $\mathrm{pH}$ decreases in the $\mathrm{pH}$ range 2.6-7.0. This results in more deposition of PLGA and thicker films at $\mathrm{pH} 4.0$ than at $\mathrm{pH}$ 7.0; PLL/PLGA multilayer nanofilms assembled at pH 4.0 presented a higher absorbance and higher thickness than those deposited at $\mathrm{pH} 7.0$ (Figure 2). When incubating 
(a)

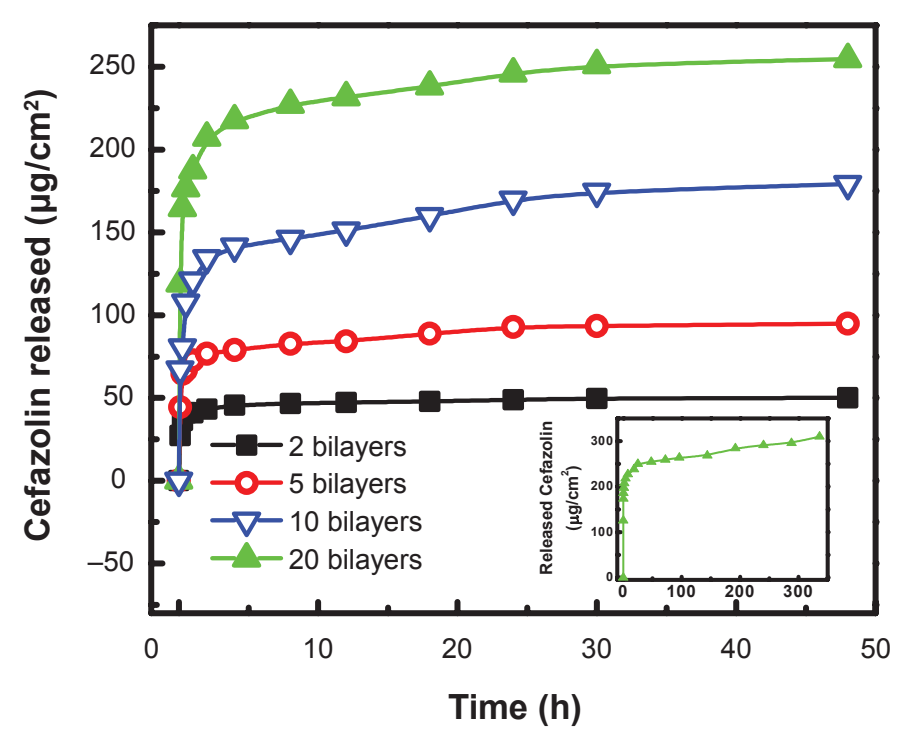

(b)

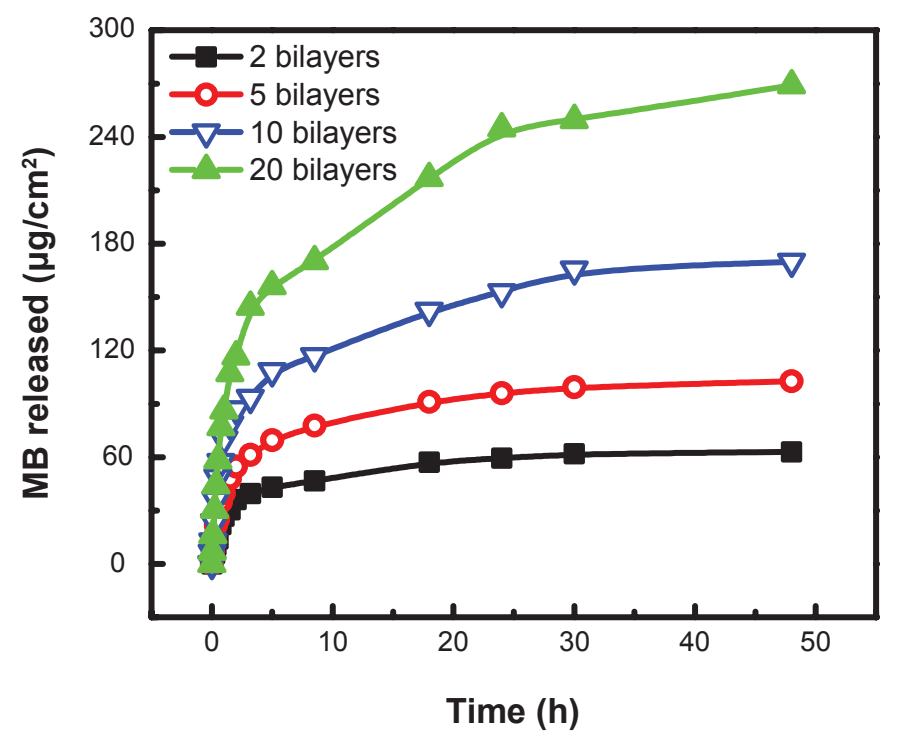

Figure 8 Release profiles of (a) cefazolin and (b) MB from polypeptide multilayer nanofilms. Both cefazolin and MB were released at pH 7.0. Cefazolin was released from PLL/PLGA multilayer nanofilms that were assembled at $\mathrm{pH} 10.0$ and loaded with cefazolin at pH 7.0 for 20 min. MB was released from PLL/PLGA multilayer nanofilms that were prepared at $\mathrm{pH} 4.0$ and loaded with $\mathrm{MB}$ at $\mathrm{pH} 7.0$ for $20 \mathrm{~min}$. The inset presents the release, up to $336 \mathrm{~h}$, of cefazolin from (PLL/PLGA) 20 nanofilms.

Abbreviations: MB, methylene blue; PLGA, poly-L-glutamic acid; PLL, poly-L-lysine.

PLL/PLGA multilayer nanofilms prepared at $\mathrm{pH} 4.0$ in a drug solution of $\mathrm{pH}$ 7.0, where both PLL and PLGA are highly ionized, the uncharged side chains of PLGA in the nanofilms become negatively charged thereby generating binding sites for positively-charged drug molecules.
In the $\mathrm{pH}$ range 4.0-7.0, the higher the drug solution $\mathrm{pH}$, the more binding sites are available and the more drugs can be captured. Therefore the capture of drugs is $\mathrm{pH}$-dependent. Meanwhile, the release of drugs from PLL/PLGA multilayer nanofilms is also $\mathrm{pH}$-dependent. When drug-loaded 
(a)

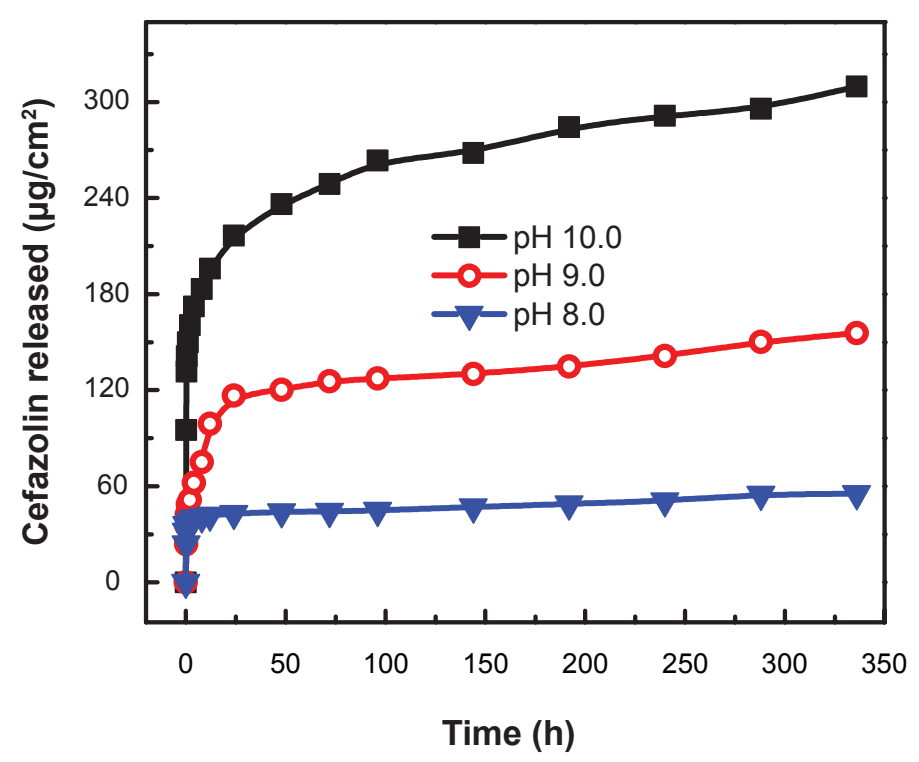

(b)

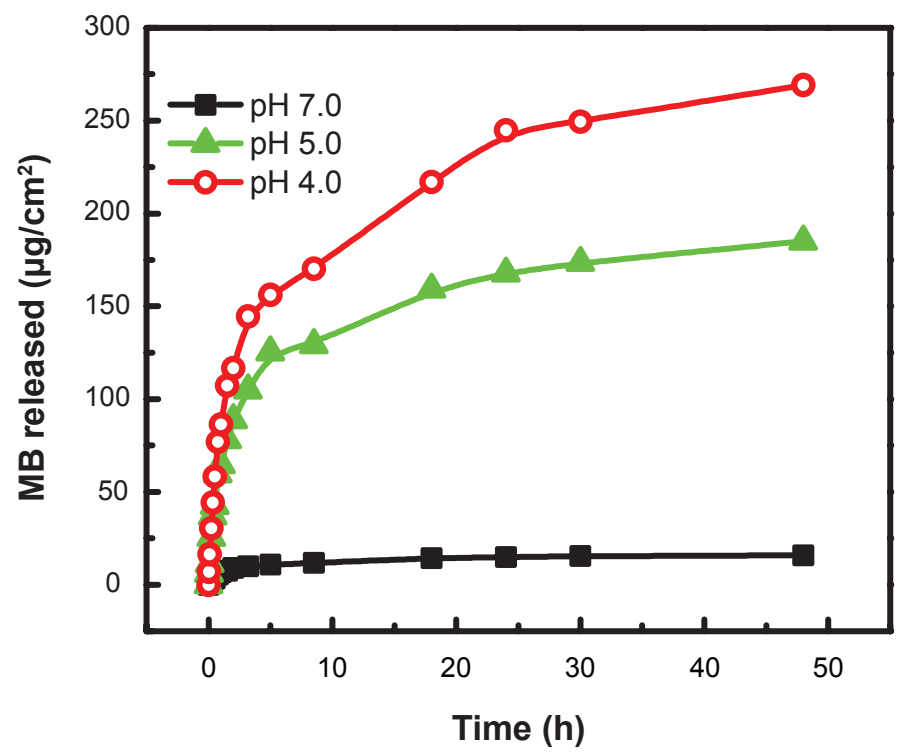

Figure 9 Effects of $\mathrm{pH}$ at which polypeptide multilayer nanofilms were assembled on release of (a) cefazolin and (b) MB. Both cefazolin and MB were released at pH 7.0. Cefazolin was released from (PLL/PLGA) ${ }_{20}$ nanofilms that were loaded with cefazolin at pH 7.0 for $20 \mathrm{~min}$. MB was released from (PLL/PLGA) ${ }_{20}$ nanofilms that were loaded with $\mathrm{MB}$ at $\mathrm{pH} 7.0$ for $20 \mathrm{~min}$.

Abbreviations: MB, methylene blue; PLGA, poly-L-glutamic acid; PLL, poly-L-lysine.

PLL/PLGA multilayer nanofilms serve in an environment of $\mathrm{pH}$ (eg, $\mathrm{pH}$ 4.0) lower than the $\mathrm{pH}$ (eg, $\mathrm{pH}$ 7.0) at which the drugs are loaded, the net charge of PLGA reverses and PLGA becomes less ionized. This leads to weakening of the interactions between the positively-charged drug molecules and the negatively-charged PLGA molecules. As a result, drugs are released from PLL/PLGA multilayer nanofilms.

Similarly (Figure 12 bottom part), PLL/PLGA multilayer nanofilms assembled at a high $\mathrm{pH}$ (eg, $\mathrm{pH} 10.0)$ contain partiallyionized and coil-structured PLL. This allows the PLL/PLGA 


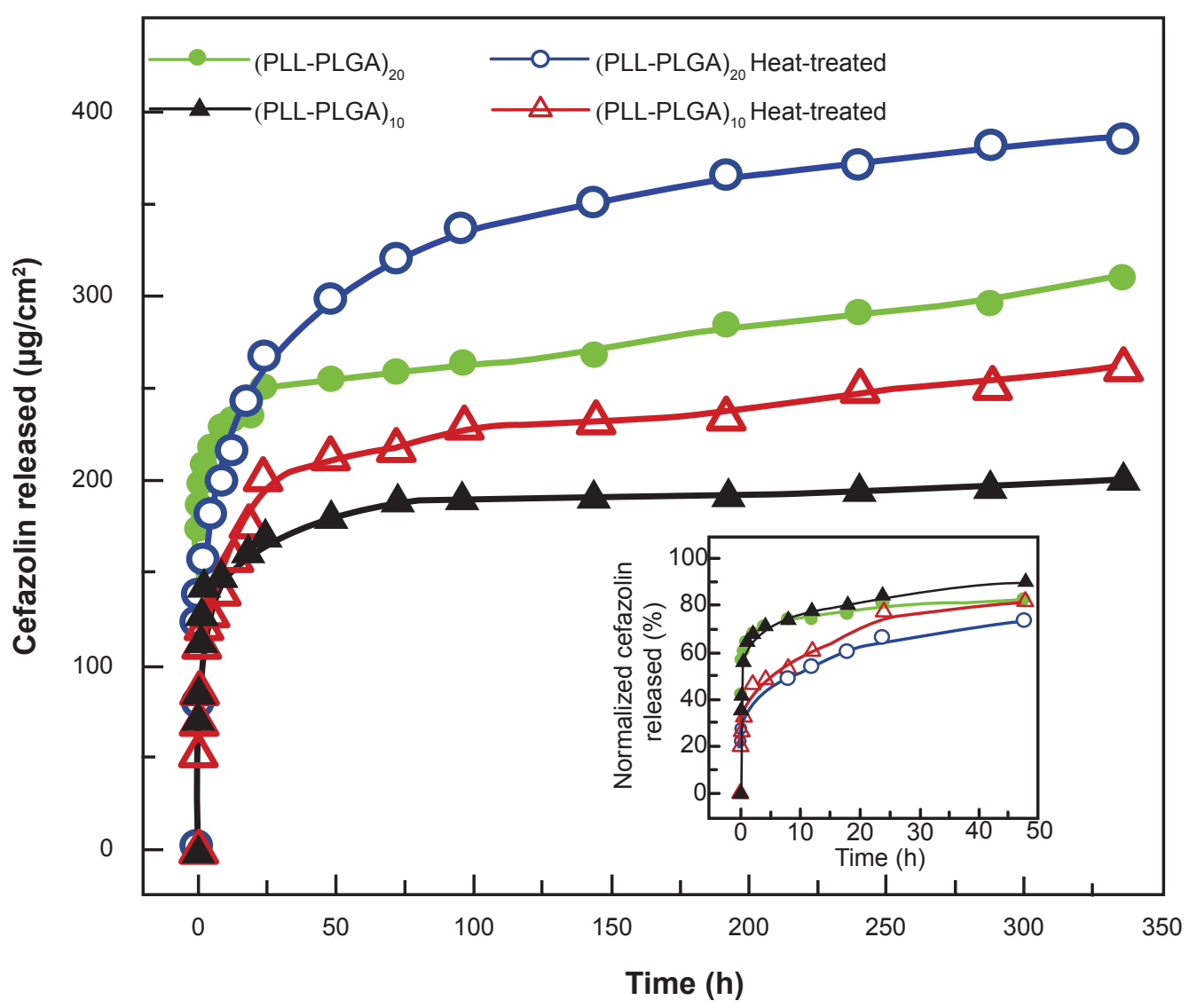

Figure 10 Effects of heat-treatment on cefazolin release from polypeptide multilayer nanofilms. Cefazolin was released at pH 7.0. Cefazolin-containing (PLL/PLGA) ${ }_{10}$ and (PLL/PLGA) ${ }_{20}$ nanofilms were assembled at pH 10.0 and loaded with cefazolin at pH 7.0 for $20 \mathrm{~min}$. The inset shows the release in percentage.

Abbreviations: PLGA, poly-L-glutamic acid; PLL, poly-L-lysine.

multilayer nanofilms the capability to load negatively-charged drugs at $\mathrm{pH} 7.0$, and drug release can be induced in an environment of a higher $\mathrm{pH}$, eg, $\mathrm{pH} 8.0,9.0$, or 10.0 .

\section{Tunable loading of charged drugs in polypeptide multilayer nanofilms}

The $\mathrm{pH}$ at which polypeptide multilayer nanofilms are prepared may influence the amount of polymers deposited thereby influencing subsequent drug loading. Figure $3 \mathrm{a}$ shows that the drug-loading capacity of (PLL/PLGA) nanofilms prepared at $\mathrm{pH} 10.0$ was approximately six times that of nanofilms assembled at $\mathrm{pH}$ 7.0. The higher loading of cefazolin in those nanofilms prepared at $\mathrm{pH} 10.0$ was due to the higher amount of PLL assembled (corresponding to the higher absorbance and increased thickness at $\mathrm{pH} 10.0$ in Figure 2) and the more ionization of PLL as the $\mathrm{pH}$ changes from film preparation at $\mathrm{pH} 10.0$ to drug loading at $\mathrm{pH} 7.0$, compared to those nanofilms prepared at $\mathrm{pHs} 9.0$ and 7.0. As a result, binding sites on PLL molecules for negatively-charged drug molecules were created as the $\mathrm{pH}$ shifts from the film preparation $\mathrm{pH}$ to the drug-loading $\mathrm{pH}$ and more binding sites on PLL would be available at a lower $\mathrm{pH}$ in the $\mathrm{pH}$ range 7.0-10.0. This is consistent with the increased loading of cefazolin in PLL/PLGA multilayer nanofilms assembled at pH 10.0 than at pHs 9.0 and 7.0 (Figure 3a).

Similarly, (PLL/PLGA) ${ }_{20}$ nanofilms assembled at a lower $\mathrm{pH}$ in the $\mathrm{pH}$ range 4.0-7.0 would have more PLGA deposited, corresponding to higher absorbance and higher thickness at pH 4.0 than at $\mathrm{pH} 7.0$ in Figure 2. This thereby leads to more binding sites on PLGA for positively-charged drug molecules when the drugs are loaded at $\mathrm{pH}$ 7.0. As shown in Figure 3b, the (PLL/PLGA) ${ }_{20}$ nanofilms formed at a lower $\mathrm{pH}$ in the $\mathrm{pH}$ range of 4.0-7.0 showed higher MB loading. Therefore, polypeptide multilayer nanofilms assembled at different $\mathrm{pHs}$ had various capacities for drug loading after film formation.

Meanwhile, the $\mathrm{pH}$ at which drugs are loaded could also be used to tune drug loading in polypeptide multilayer nanofilms. 
(a)

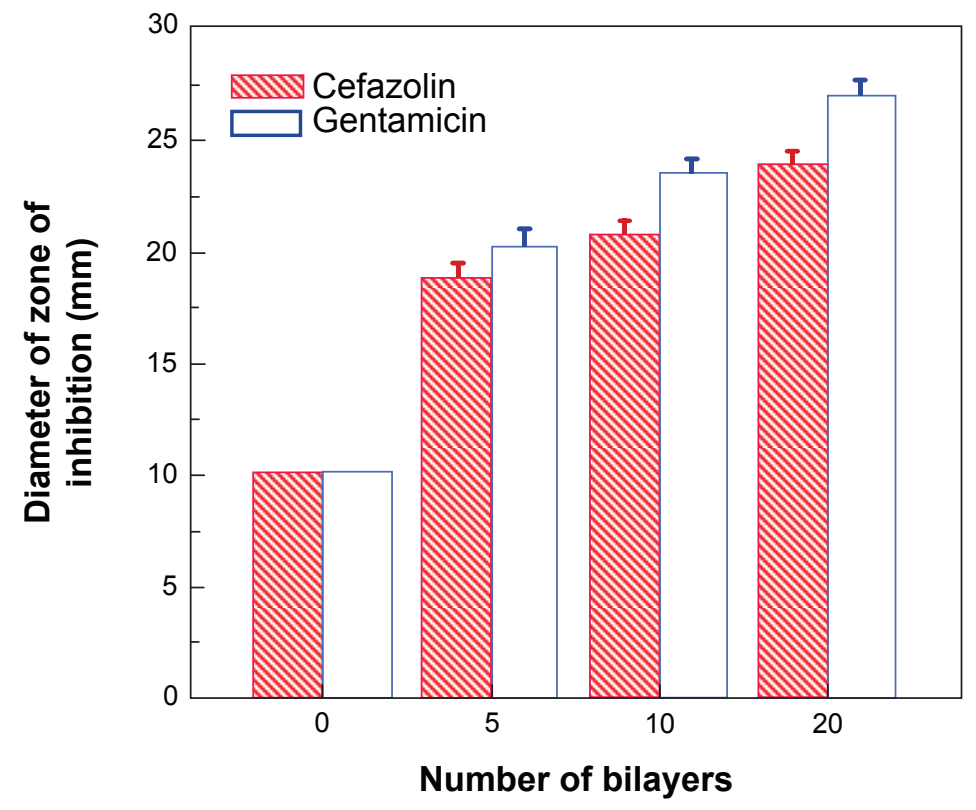

(b)

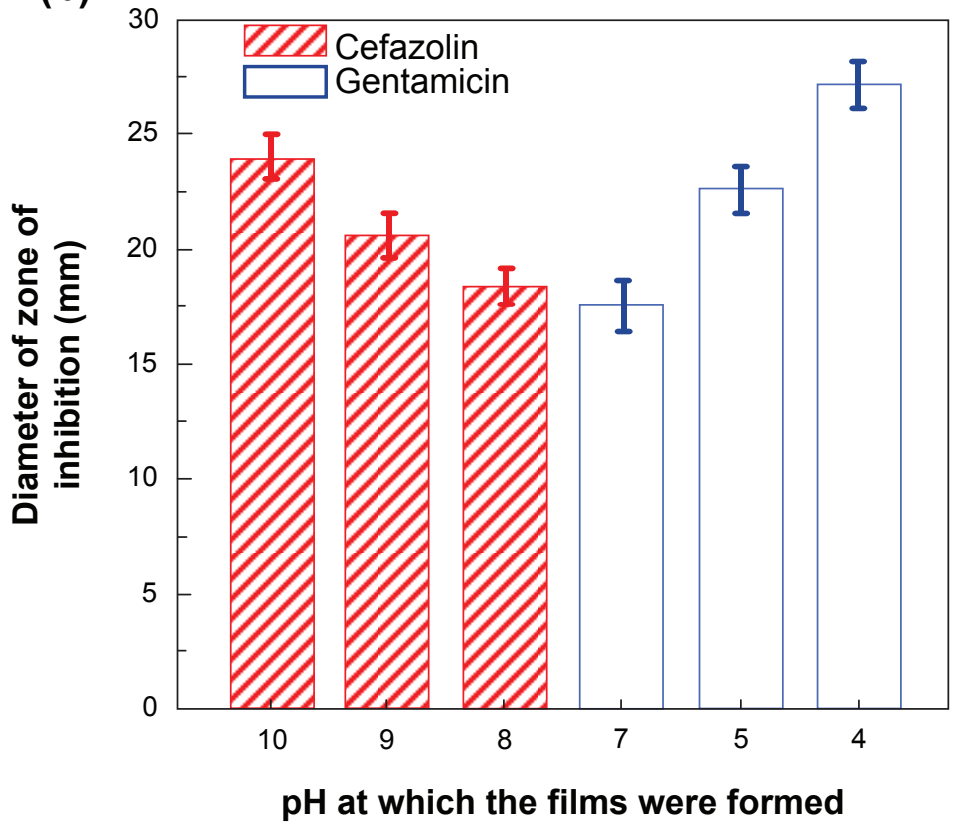

Figure II a) Diameter of zone of inhibition vs number of film bilayers. Cefazolin-containing PLL/PLGA nanofilms were assembled at pH I0.0 and loaded with cefazolin at $\mathrm{pH} 7.0$ for 20 min. Gentamicin-containing PLL/PLGA nanofilms were assembled at pH 4.0 and loaded with gentamicin at pH 7.0 for 20 min. b) Diameter of zone of inhibition vs. $\mathrm{pH}$ at which drug-containing (PLL/PLGA) ${ }_{20}$ nanofilms were assembled. (PLL/PLGA) ${ }_{20}$ nanofilms were loaded with cefazolin or gentamicin at $\mathrm{pH} 7.0$ for 20 min. The diameter of control samples was $10.0 \mathrm{~mm}$.

Abbreviations: PLGA, poly-L-glutamic acid; PLL, poly-L-lysine.

As shown in Figure 4, (PLL/PLGA) $)_{20}$ nanofilms prepared at $\mathrm{pH} 10.0$ showed different capacities for loading negativelycharged cefazolin in the $\mathrm{pH}$ range 7.0-10.0, and (PLL/ PLGA) $)_{20}$ nanofilms prepared at $\mathrm{pH} 4.0$ allowed the tuning of positively-charged $\mathrm{MB}$ loading in the $\mathrm{pH}$ range 4.0-7.0. More side chains of PLL, assembled at $\mathrm{pH} 10.0$, became ionized at a lower drug-loading $\mathrm{pH}$ in the $\mathrm{pH}$ range $7.0-10.0$, therefore more binding sites were available for negatively-charged drug molecules and more cefazolin was captured at $\mathrm{pH} 7.0$ than at pHs 8.0, 9.0, and 10.0 (Figure 4). At pH 10.0, no absorbance for cefazolin was detected in the nanofilms, because there was no $\mathrm{pH}$ shift between film preparation and drug loading and 
$\mathrm{pH}<7.0$ (eg, 4.0)
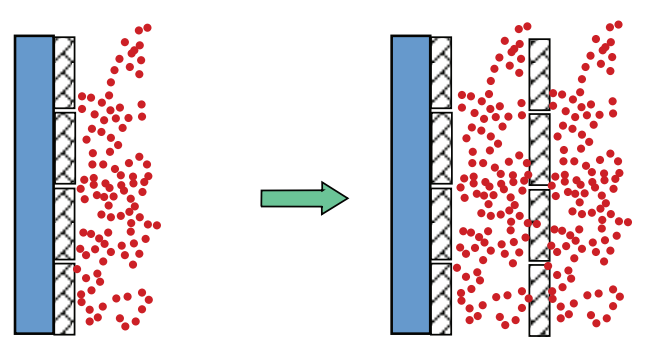

$\bullet \bullet \bullet \quad$ Coating formation
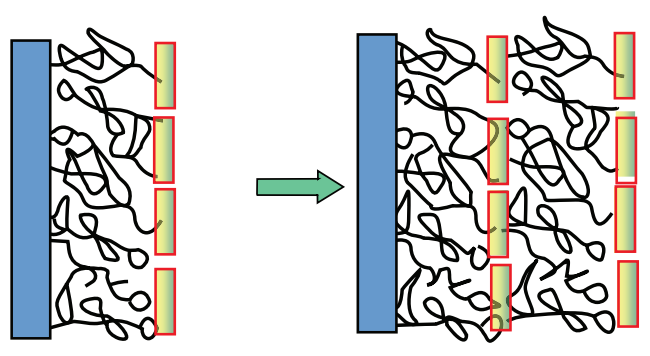

$\mathrm{pH}>7.0(\mathrm{eg}, 10.0)$

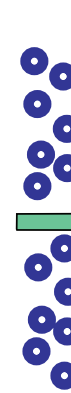

$\mathrm{pH} 7.0$

$\mathrm{pH}<7.0(\mathrm{eg}, 4.0)$

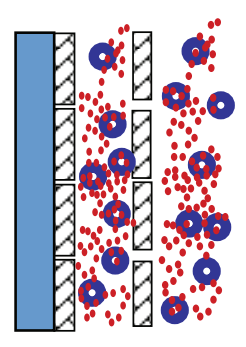

Drug loading

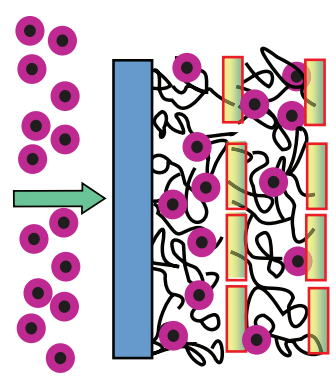

$\mathrm{pH} 7.0$

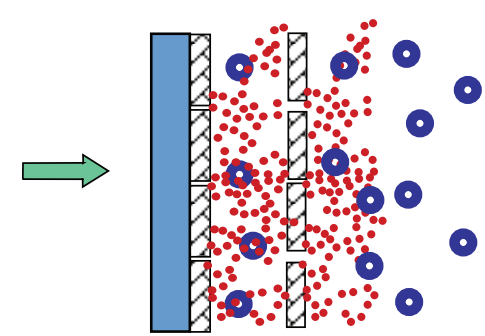

$\bullet \bullet \bullet \bullet$ Drug release

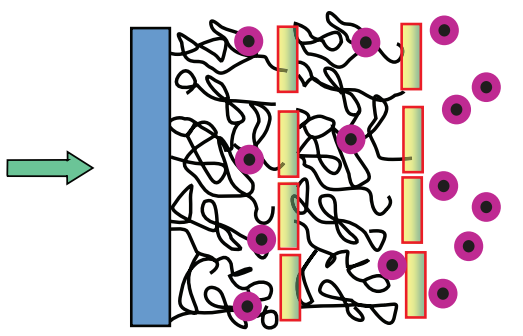

$\mathrm{pH}>7.0(\mathrm{eg}, 10.0)$

Figure 12 Mechanisms of $\mathrm{pH}$-dependent drug capture and release from polypeptide multilayer nanofilms. PLL/PLGA multilayer nanofilms are assembled at a pH (eg, $\mathrm{pH} 4.0$ or 10.0) away from the drug-loading $\mathrm{pH}$, followed by incubating in a drug (eg, gentamicin, $\mathrm{MB}$, or cefazolin) solution of $\mathrm{pH} 7.0$ and releasing the captured drugs by altering the $\mathrm{pH}$ of releasing media.

Key: 目 stretched PLL, coiled PLL, 目 stretched PLGA, coiled PLGA, $\bullet$ positively-charged drug, $\bullet$ negatively-charged drug.

Abbreviations: MB, methylene blue; PLGA, poly-L-glutamic acid; PLL, poly-L-lysine.

no binding sites were created; as a result, no cefazolin was captured in the nanofilms. This may mean that the driving force of drug loading in PLL/PLGA multilayers is mainly electrostatic attraction. Similarly, the drug solution $\mathrm{pH}$ also influenced the loading of positively-charged $\mathrm{MB}$, where the PLL/PLGA nanofilms were assembled at $\mathrm{pH}$ 4.0. The change in ionization of PLGA led to the capture of more positivelycharged $\mathrm{MB}$ at $\mathrm{pH} 7.0$ than at $\mathrm{pHs} 4.0$ and 5.0 (Figure 4). The limited loading of $\mathrm{MB}$ at $\mathrm{pH} 4.0$ might be related to the interaction of MB with the outermost layer, ie, PLGA, of the (PLL/PLGA) $)_{20}$ nanofilm.

Using the electrostatic layer-by-layer self-assembly technique, we can also control the amount of polymers deposited and tune the subsequent drug loading by manipulating the number of deposition bilayers. Figure 2 shows that the absorbance and thickness, and thereby the amount of polypeptides deposited, increased with increasing deposition bilayers. The increase in polypeptide deposition could lead to an increase in binding sites and drug loading when the $\mathrm{pH}$ shifts from the deposition $\mathrm{pH}$ to the drug-loading $\mathrm{pH}$. As a result, the amounts of cefazolin and MB loaded in PLL/PLGA multilayer nanofilms increased approximately linearly with increasing deposition bilayers (Figure 5a).
In addition, heat-treatment after film formation may influence drug loading. We found that heat-treatment after multilayer film formation led to an increase $(\sim 30 \%$, Figure 5b) in cefazolin loading; however, the reason for the increase is unknown and will be studied in the future. In the literature, heat-treatment led to the formation of crosslinkings between polyelectrolyte multilayers due to the formation of amide bonds from carboxylic and amine groups within polyelectrolyte multilayers, ${ }^{35}$ and the swelling properties of heat-treated films changed significantly over a wide $\mathrm{pH}$ range. ${ }^{36}$

\section{Tunable drug release from polypeptide multilayer nanofilms}

In previous studies, drug release from polyelectrolyte multilayer films was mainly controlled by manipulating the permeability and degradation of the films. In our study, PLL/ PLGA multilayer nanofilms were stable in aqueous solutions and could tolerate $\mathrm{pH}$ shifts in our drug loading and release processes. The release of drug molecules from polypeptide multilayer nanofilms is mainly due to the change of interaction between drug molecules and polypeptide nanofilms, and drug diffusion. 
By changing the interaction between drug molecules and PLL/PLGA multilayer film components, one can tune drug release. As shown in Figure 12, when there is a pH shift from drug loading to drug release, the interaction between the drug molecules and the corresponding oppositely-charged polypeptides changes and drugs can be released. Cefazolin and MB were loaded at $\mathrm{pH} 7.0$ and more cefazolin was released at $\mathrm{pH} 10.0$ than at a lower $\mathrm{pH}$ in the $\mathrm{pH}$ range $7.0-10.0$. More MB was released at $\mathrm{pH} 4.0$ than at a higher $\mathrm{pH}$ in the $\mathrm{pH}$ range $4.0-7.0$ (Figure 7), due to the change in the interaction between the drug molecules and the corresponding oppositely-charged polypeptides. For instance, as the $\mathrm{pH}$ changed from the drug loading $(\mathrm{pH} 7.0)$ to a higher drug release $\mathrm{pH}$ in the $\mathrm{pH}$ range 7.0-10.0, more binding sites of PLL were reversed and became uncharged; therefore, more cefazolin was released from the nanofilms.

Moreover, the history of polypeptide multilayer nanofilms had a significant impact on the amount of drugs released. Drug release could be tuned by controlling the number of film layers, the $\mathrm{pH}$ at which the nanofilms were prepared, and post-preparation heat-treatment. It was found that more drugs (cefazolin and $\mathrm{MB}$ ) were released from PLL/PLGA multilayer nanofilms with more bilayers (Figure 8). More cefazolin was released from (PLL/PLGA) 20 nanofilms assembled at $\mathrm{pH} 10.0$ than those assembled at a lower $\mathrm{pH}$ in the $\mathrm{pH}$ range 7.0-10.0, and more $\mathrm{MB}$ was released from (PLL/PLGA $)_{20}$ nanofilms assembled at $\mathrm{pH} 4.0$ than those nanofilms prepared at a higher $\mathrm{pH}$ in the $\mathrm{pH}$ range 4.0-7.0 (Figure 9). The higher drug release was probably associated with the corresponding higher drug loading in the PLL/PLGA multilayer nanofilms under those conditions (Figure 3). The post-preparation heat-treatment also had some effect on drug release, as it seemed that heat-treatment slowed drug release compared to the control samples (Figure 10 inset). This was likely related to the possible crosslinking formation and the reduced swelling of polyelectrolyte multilayers in aqueous media after heat-treatment. ${ }^{36}$ The higher release amounts of cefazolin after the burst release period was because of higher amounts of cefazolin loaded in the nanofilms after heat-treatment (Figure 5b).

\section{In vitro antibacterial activity against $S$. aureus}

Quantitative assessment of the therapeutic activity of antibiotic-loaded polypeptide multilayer nanofilms was conducted. The diameter of a zone of inhibition provides a quantitative measure of the amount of in vitro active antibiotic (eg, cefazolin and gentamicin) released and diffused into the agar plates. Figure 11 shows that PLL/PLGA multilayer nanofilms containing cefazolin and gentamicin presented large zones of inhibition, and those samples without antibiotics had no antibacterial effects. The zone of inhibition became larger with increasing number of bilayers; this is because more drugs (eg, cefazolin) were loaded and subsequently released from the PLL/PLGA multilayer nanofilms with more bilayers (Figure 5a). The difference in the sizes of zone of inhibition in (PLL/PLGA) ${ }_{20}$ nanofilms assembled at different $\mathrm{pHs}$ was also related to the different amounts of antibiotics loaded and released from the PLL/PLGA multilayer nanofilms. The more antibiotics loaded, the bigger the zone of inhibition. Figure 3 shows that the (PLL/PLGA) ${ }_{20}$ nanofilms formed at different $\mathrm{pHs}$ possessed different drug-loading capacities, and more cefazolin was loaded at $\mathrm{pH} 10.0$ than at $\mathrm{pHs} 9.0$ and 7.0. As a result, a larger zone of inhibition was observed in cefazolin loaded (PLL/PLGA) 20 nanofilms assembled at $\mathrm{pH} 10.0$ than at $\mathrm{pHs} 9.0$ and 7.0.

Our studies showed that stainless steel discs coated with antibiotic-loaded polypeptide multilayer nanofilms exhibited in vitro antibacterial activity against $S$. aureus. Stainless steel is one of the commonly used metal implants in orthopedics and $S$. aureus is the most common source of osteomyelitis and septic arthritis. ${ }^{37,38}$ Therefore, the developed antibioticloaded polypeptide multilayer nanofilms have the potential to prevent orthopaedic device-associated infection, and further studies will be carried out to investigate the efficacy of such antibiotic-loaded nanofilms in preventing infection in vivo in an open fracture rat model we recently developed. ${ }^{39}$

\section{Conclusions}

A multilayer self-assembly technology was applied to construct biodegradable polypeptide multilayer nanofilms made of PLL and PLGA, which are weak polyelectrolytes that enable the fine tuning of drug loading and release after film formation. Our studies showed that the loading kinetics of gentamicin and MB, both positively-charged, were very similar. The loading and release of both negatively- and positively-charged drug molecules (eg, cefazolin, gentamicin, and MB) could be tuned by several variables during and after the film preparation. Such variables include the number of deposition layers, $\mathrm{pH}$ of film preparation, and post-preparation heat-treatment. The loading of drugs (eg, cefazolin and MB) increased approximately linearly with an increasing number of layers, and heat-treatment before drug loading enhanced the drug-loading capacity. The $\mathrm{pH}$ of film preparation also significantly altered the film formation including surface morphology. In addition, the drug-loading $\mathrm{pH}$ and the incubation time in the drug solution could be used 
to tune the amount of drugs that could be loaded, and the $\mathrm{pH}$ of an application environment also had a significant impact on drug release. The developed antibiotic-loaded polypeptide multilayer nanofilms presented tunable antibacterial properties and potentially have significant applications in medicine, eg, antibacterial drug delivery systems for preventing biomedical device-associated infection.

\section{Acknowledgments}

The authors received financial support from National Science Foundation (NSF), West Virginia University Program to Stimulate Competitive Research (WVU PSCoR), West Virginia University Senate Grant, and National Aeronautics and Space Administration West Virginia Experimental Program to Stimulate Competitive Research (NASA WV EPSCoR). We also acknowledge the assistance of Rajiv Bhattarai (Davis and Elkins College, Elkins, WV) for film stability tests and staff support by Vincent Kish, Suzanne Smith, and Nina Clovis.

\section{References}

1. Garvin K, Feschuk C. Polylactide-polyglycolide antibiotic implants. Clin Orthop Relat Res. 2005;437:105-110.

2. Zalavras CG, Patzakis MJ, Holtom PD, Sherman R. Management of open fractures. Infect Dis Clin North Am. 2005;19:915-929.

3. Schmidmaier G, Lucke M, Wildemann B, Haas NP, Raschke M. Prophylaxis and treatment of implant-related infections by antibioticcoated implants: a review. Injury. 2006;37:S105-S112.

4. Murray CK, Hsu JR, Solomkin JS, Keeling JJ, Andersen RC, Ficke JR, Calhoun JH. Prevention and management of infections associated with combat-related extremity injuries. J Trauma. 2008;64(3 Suppl):S239-S251.

5. Langer R. New methods of drug delivery. Science. 1990;249:1527-1533.

6. Sun YM, Chang CC, Huang WF, Liang HC. Fluidized-bed spray coated porous hydrogel beads for sustained release of diclofenac sodium. J Control Release. 1997;47:247-260.

7. Uhrich KE, Cannizzaro SM, Langer RS, Shakesheff KM. Polymeric systems for controlled drug release. Chem Rev. 1999;99:3181-3198.

8. Acharya G, Park K. Mechanisms of controlled drug release from drugeluting stents. Adv Drug Del Rev. 2006;58:387-401.

9. Muller-Buschbaum P, Gebhardt R, Maurer E, Bauer E, Gehrke R, Doster W. Thin casein films as prepared by spin-coating: Influence of film thickness and of pH. Biomacromolecules. 2008;7:1773-1780.

10. Antoci V, Adams CS, Parvizi J, Ducheyne P, Shapiro IM, Hickok NJ. Covalently attached vancomycin provides a nanoscale antibacterial surface. Clin Orthop Relat Res. 2007;461:81-87.

11. Decher G. Fuzzy nanoassemblies: toward layered polymeric multicomposites. Science. 1997;277:1232-1237.

12. Lynn DM. Layers of opportunity: nanostructured polymer assemblies for the delivery of macromolecular therapeutics. Soft Matt. 2006;2:269-273.

13. De Geest BG, Sanders NN, Sukhorukov GB, Demeester J, De Smedt SC. Release mechanisms for polyelectrolyte capsules. Chem Soc Rev. 2007;36:636-649.

14. Scranton AB, Rangarajan B, Klier J. Biomedical applications of polyelectrolytes. Adv Polym Sci. 1995;122:1-54.

15. Cai KY, Rechtenbach A, Hao JY. Polysaccharide-protein surface modification of titanium via a layer-by-layer technique: Characterization and cell behavior aspects. Biomaterials. 2005;26:5960-5971.

16. Quinn JF, Caruso F. Thermoresponsive nanoassemblies: Layerby-layer assembly of hydrophilic-hydrophobic alternating copolymers. Macromolecules. 2005;38:3414-3419.
17. Chluba J, Voegel JC, Decher G, Erbacher P, Schaaf P, Ogier J. Peptide hormone covalently bound to polyelectrolytes and embedded into multilayer architectures conserving full biological activity. Biomacromolecules. 2001;2:800-805.

18. Serizawa T, Yamaguchi M, Akashi M. Alternating bioactivity of polymeric layer-by-layer assemblies: Anti- vs procoagulation of human blood. Biomacromolecules. 2002;3:724-731.

19. Jessel N, Atalar F, Lavalle P, et al. Bioactive coatings based on a polyelectrolyte multilayer architecture functionalized by embedded proteins. Adv Mater. 2003;15:692-695.

20. Amiji M, editor. Polymeric gene delivery: Principles and applications. New York, NY: CRC Press;2004. p. 227-241.

21. Schultz P, Vautier D, Richert L, et al. Polyelectrolyte multilayers functionalized by a synthetic analogue of an anti-inflammatory peptide, alpha-MSH, for coating a tracheal prosthesis. Biomaterials. 2005;26:2621-2630.

22. Zhong Y, Whittington CF, Haynie DT. Stimulated release of small molecules from polyelectrolyte multilayer nanocoatings. Chem Commun. 2007;14:1415-1417.

23. Mendelsohn JD, Barrett CJ, Chan VV, Pal AJ, Mayes AM, Rubner MF. Fabrication of microporous thin films from polyelectrolyte multilayers. Langmuir. 2000;16:5017-5023.

24. Berg MC, Zhai L, Cohen RE, Rubner MF. Controlled drug release from porous polyelectrolyte multilayers. Biomacromolecules. 2006;7:357-364.

25. Qi B, Tong X, Zhao Y. Layer-by-layer assembly of two different polymer micelles with polycation and polyanion coronas. Macromolecules. 2006;39:5714-5719.

26. Schneider A, Vodouhe C, Richert L, et al. Multifunctional polyelectrolyte multilayer films: Combining mechanical resistance, biodegradability, and bioactivity. Biomacromolecules. 2007;8:139-145.

27. Wang L, Wang X, Xu MF, Chen DD, Sun JQ. Layer-by-layer assembled microgel films with high loading capacity: Reversible loading and release of dyes and nanoparticles. Langmuir. 2008;24:1902-1909.

28. Arici MK, Sumer Z, Guler C, Elibol O, Saygi G, Cetinkaya S. In vitro potency and stability of fortified ophthalmic antibiotics. Aust $N Z J$ Ophthalmol. 1999;27:426-430.

29. Chung AJ, Rubner MF. Methods of loading and releasing low molecular weight cationic molecules in weak polyelectrolyte multilayer films. Langmuir. 2002;18:1176-1183.

30. Chuang HF, Smith RC, Hammond PT. Polyelectrolyte multilayers for tunable release of antibiotics. Biomacromolecules. 2008;9:1660-1668.

31. Sherertz RJ, Forman DM, Solomon DD. Efficacy of dicloxacillin-coated polyurethane catheters in preventing subcutaneous Staphylococcus aureus infection in mice. Antimicrob Agents Chemother. 1989;33:1174-1178.

32. Jiang B, Li B. Polypeptide nanocoatings for preventing dental and orthopaedic device-associated infection. $J$ Biomed Mater Res B. 2009;88:332-338.

33. Zimmermann R, Kratzmuller T, Erickson D, Li DQ, Braun HG, Werner C. Ionic strength-dependent $\mathrm{pK}$ shift in the helix-coil transition of grafted poly(l-glutamic acid) layers analyzed by electrokinetic and ellipsometric measurements. Langmuir. 2004;20:2369-2374.

34. Hahn SK, Hoffman AS. Preparation and characterization of biocompatible polyelectrolyte complex multilayer of hyaluronic acid and poly-L-lysine. Int J Biol Macromol. 2005;37:227-231.

35. Harris JJ, DeRose PM, Bruening ML. Synthesis of passivating, nylonlike coatings through cross-linking of ultrathin polyelectrolyte films. J Am Chem Soc. 1999;121:1978-1979.

36. Tong WJ, Gao CY. Stable microcapsules assembled stepwise from weak polyelectrolytes followed by thermal crosslinking. Polym Adv Technol. 2005; $16: 827-833$.

37. Goldenberg DL. Septic arthritis. Lancet. 1998; 351:197-202.

38. Marriott I, Gray DL, Tranguch SL, et al. Osteoblasts express the inflammatory cytokine interleukin-6 in a murine model of Staphylococcus aureus osteomyelitis and infected human bone tissue. Am J Pathol. 2004;164:1399-1406.

39. Li B, Jiang B, Boyce B, Lindsey B. Oral presentation, Local IL-12 incorporated in nanocoatings promising for preventing open fracture associated infection. San Francisco, CA: Orthopaedic Research Society (ORS) Annual Meeting; March 2008. 
